\title{
Hemicolectomía derecha laparoscópica por puerto único para cáncer de colon*
}

\author{
Drs. FRANCISCO LÓPEZ K. ${ }^{1}$, RODRIGO CÁPONA P. ${ }^{1}$, CLAUDIO HEINE T. ${ }^{1}$, \\ CLAUDIO WAINSTEIN G. ${ }^{1}$, NICOLÁS MÁRQUEZ A. ${ }^{1}$ \\ 1 Unidad de Coloproctología de Clínica Las Condes, Santiago, Chile.
}

\begin{abstract}
Single-port access right laparoscopic hemicolectomy for colon cancer

A new laparoscopic technique allows a peritoneal access through a single umbilical port with less abdominal wall trauma and pain. We report a 64 years old male with a right colon adenocarcinoma, detected during a routine colonoscopy. The patient was subjected to a right laparoscopic hemicolectomy through a single SILS ${ }^{\circledR}$ Multiple Instrument Access Port, that can accommodate up to three instruments introduced through a $3 \mathrm{~cm}$ paraumbilical incision. The surgical procedure was uneventful and the patient had a good postoperative evolution. The pathological study of the surgical piece confirmed the presence of an adenocarcinoma with invasion of the submucosa and without lymph node involvement.
\end{abstract}

Key words: Single port, laparoscopic surgery, hemicolectomy.

\section{Resumen}

La tendencia a una menor invasión en el acto quirúrgico ha sido un objetivo desde los inicios de la cirugía y en la actualidad se buscan continuamente caminos que cumplan este principio. En el último año han surgido publicaciones de una nueva técnica laparoscópica a través de un acceso umbilical único que permitiría disminuir el trauma de la pared abdominal, el dolor postoperatorioy además, mejorar el resultado cosmético. El objetivo de este reporte es dar a conocer nuestra experiencia con la primera hemicolectomía derecha laparoscópica (HCDL) reportada a través de un puerto único para una neoplasia maligna de colon. Se trata de un paciente con un adenocarcinoma cecal originado en un pólipo $(<2 \mathrm{~cm})$ abordado por vía laparoscópica introduciendo el puerto único SILS (SILS ${ }^{\mathrm{TM}}$ Port, Covidien, Autosuture) con lugar para trabajar con 3 trócares a través de una incisión paraumbilical de $3 \mathrm{~cm}$, por la cual se realizó toda la disección y extracción de la pieza operatoria. La cirugía se llevó a cabo sin incidentes, respetando todos los principios oncológicos y el paciente evolucionó en forma satisfactoria. La pieza operatoria confirmó un adenocarcinoma con invasión de submucosa (SM3) sin metástasis en 30 ganglios examinados. Se concluye que la realización de una HCDL con puerto único es factible y pudiera ser segura desde el punto de vista oncológico. Tiene ventajas cosméticas evidentes y además podría disminuir la morbilidad de la pared abdominal, los costos totales quirúrgicos y eventualmente las tasas de conversión.

Palabras clave: Hemicolectomía derecha, laparoscopía, un puerto.

*Recibido el 16 de Junio de 2009 y aceptado para publicación el 28 de Julio de 2009.

Correspondencia: Dr. Francisco López K.

Lo Fontecilla 441, Santiago, Chile.

E-mail: flopez@clc.cl 


\section{Introducción}

La cirugía laparoscópica colorrectal ha experimentado un importante desarrollo en las últimas 2 décadas ${ }^{1}$, siendo actualmente el abordaje de elección en algunos centros de referencia ${ }^{2}$. Numerosas publicaciones han reportado los potenciales beneficios de la cirugía mínimamente invasiva en la patología colorrectal, tanto benigna como maligna ${ }^{3-5}$. Siguiendo la tendencia natural de la cirugía hacia procedimientos de menor invasión ${ }^{6,7}$, en los últimos años se ha desarrollado la cirugía laparoscópica por puerto único (SILS), técnica que podría cumplir con estas exigencias. La cirugía SILS consiste en realizar sólo un acceso abdominal con un puerto único ubicado en el ombligo a través del cual se introduce el instrumental y se realiza la totalidad del procedimiento, incluso la extracción de la pieza operatoria. El objetivo de este reporte es dar a conocer nuestra experiencia con la primera hemicolectomía derecha laparoscópica reportada a través de un puerto único para una neoplasia maligna de colon.

\section{Caso Clínico}

Paciente de 64 años, sin antecedentes mórbidos de importancia, que estando previamente asintomático, se realiza en Abril del 2009 una colonoscopía como parte de un programa preventivo en la que se advierte una lesión polipoidea, sésil, de aspecto velloso con una depresión central, de $12 \mathrm{~mm}$ de extensión, ubicada en el ciego frente a la válvula ileocecal. La biopsia de esta lesión resulta positiva para adenocarcinoma invasor. Dada la morfología, ubicación y riesgo de perforación, se desiste de su resección endoscópica.

Se realiza un estudio de extensión por imágenes que resulta negativo para enfermedad diseminada y se planifica para tratamiento quirúrgico. Se trata de un paciente con IMC de 26,8, sin cirugías abdominales previas, sin patologías de base y con una lesión neoplásica pequeña, que lo hacen el candidato ideal para realizar la primera hemicolectomía derecha laparoscópica por puerto umbilical único para una neoplasia maligna de colon.

\section{Técnica Quirúrgica}

Se realiza preparación de colon estándar con 4 1ts de polietilenglicol el día previo. Se coloca al paciente en posición de Lloyd Davis y se prepara el campo estéril en forma convencional. Se realiza una incisión paraumbilical de $3 \mathrm{~cm}$ para la instalación de un puerto único para tres trócares (SILS ${ }^{\mathrm{TM}}$ Port, Covidien, Autosuture) (Figuras 1 y 2). Se introduce el puerto a la cavidad abdominal y se procede a trabajar con 3 trócares (Figura 3): uno de $10 \mathrm{~mm}$ para cámara con óptica de $30^{\circ}$ y dos de $5 \mathrm{~mm}$ para una pinza angulada (Roticulator ${ }^{\mathrm{TM}}$ Endo Grasp ${ }^{\mathrm{TM}}$ $5 \mathrm{~mm}$ W/Spin Lock, Covidien, Autosuture) y elementos de disección (gancho convencional y EnSeal ${ }^{\mathrm{TM}}$ Laparoscopic Tissue Sealing Device, SurgRx, Ethicon). Se explora la cavidad abdominal sin encontrar evidencia de diseminación tumoral local ni a distancia. Con el paciente en posición de Trendelenburg y lateralización a izquierda se inicia la disección del íleon terminal por su borde inferior y luego se avanza por la corredera parietocólica derecha desprendiendo del retroperitoneo al íleon y colon derecho hasta el ángulo hepático. Posteriormente en posición de Fowler se identifica el duodeno ingresando a la transcavidad de los epiplones por su borde superior. Se efectúa disección de medial a lateral dejando el epiplón mayor con la pieza operatoria hasta llegar a la región cercana al ángulo hepático, en la cual la interposición de la vesícula biliar dificulta proseguir en la disección. Para esto se introduce una sutura de Vicryl ${ }^{\mathrm{TM}}$ 3-0
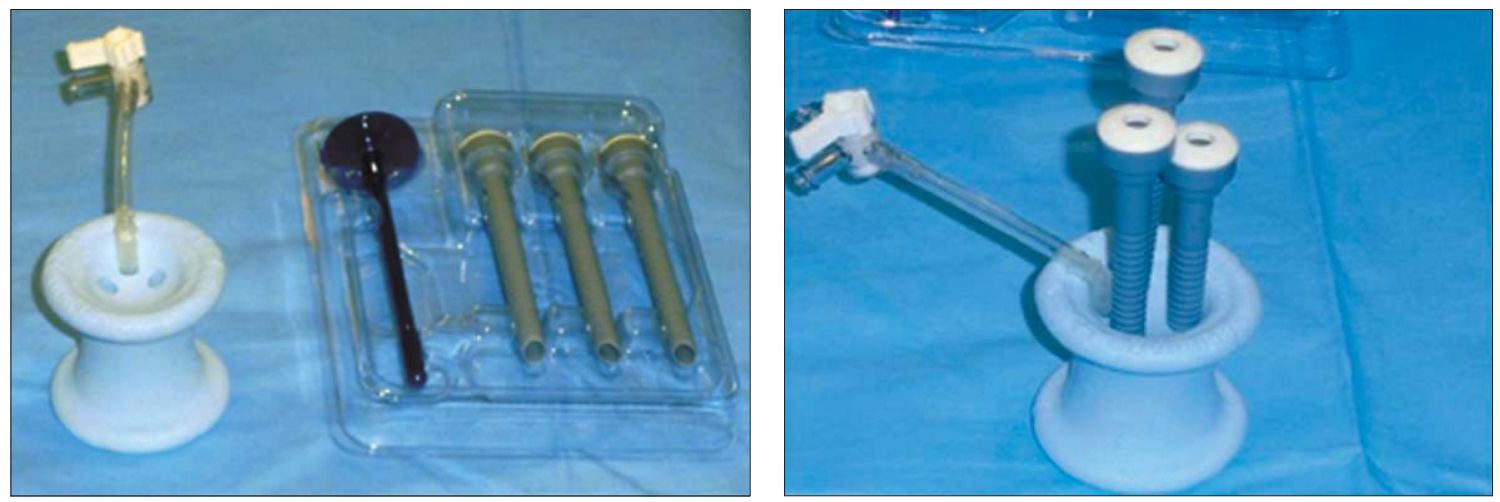

Figura 1 y 2. Puerto único para tres trócares (SILS ${ }^{\mathrm{TM}}$ Port, Covidien, Autosuture) 
en el hipocondrio derecho, con la cual se confecciona una especie de hamaca y se tracciona la vesícula hacia anterior lo que permite avanzar en la disección hasta desprender completamente el ángulo hepático del colon; posteriormente se seccionan los vasos ileocólicos por vía abierta. Se retiran los

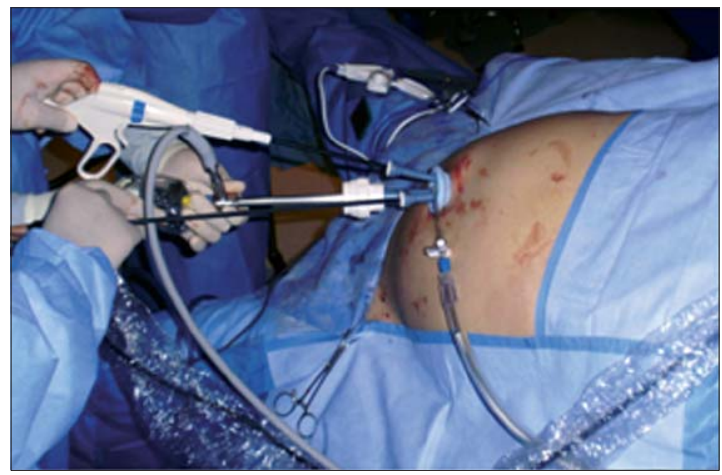

Figura 3. Instalación intraoperatoria.

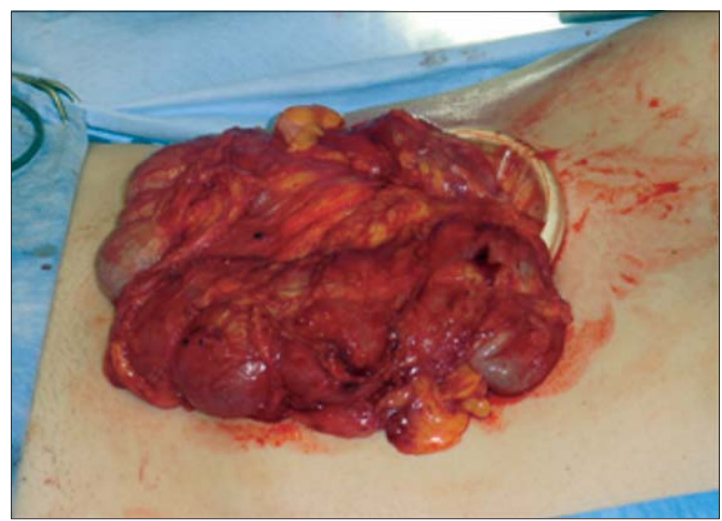

Figura 5. Pieza quirúrgica exteriorizada.

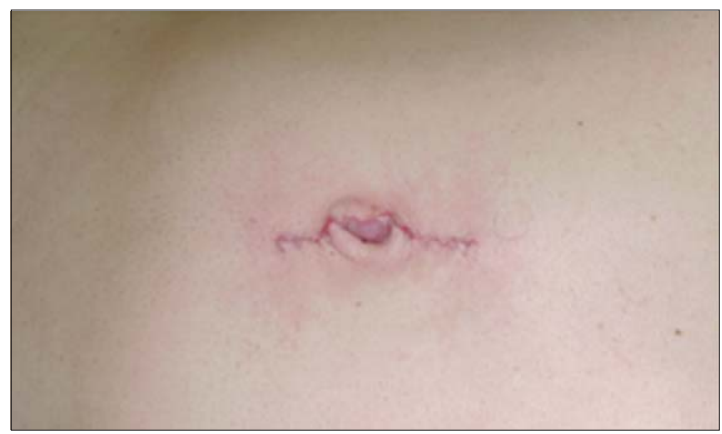

Figura 7. Laparotomía e incisión cerrada. trócares y el puerto umbilical, se amplía la incisión para la extracción de la pieza operatoria, se introduce un anillo Alexis (Alexis Wound Retractor System $^{\mathrm{TM}}$, Applied Medical) (Figura 4) por el cual se extrae el colon derecho (Figura 5). Se secciona el íleon terminal a $15 \mathrm{~cm}$ de la válvula ileocecal y se

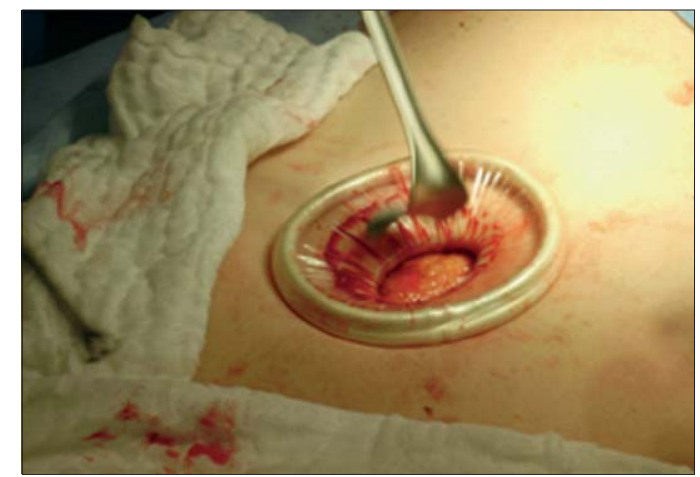

Figura 4. Anillo para extracción de pieza quirúrgica.

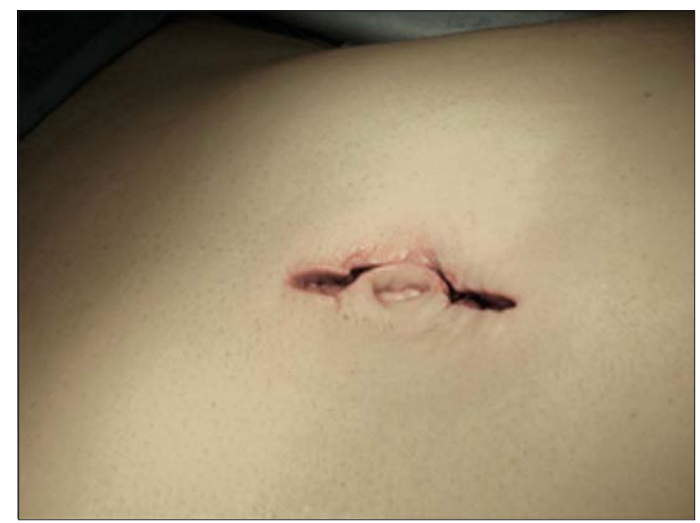

Figura 6. Laparotomía previo al cierre.

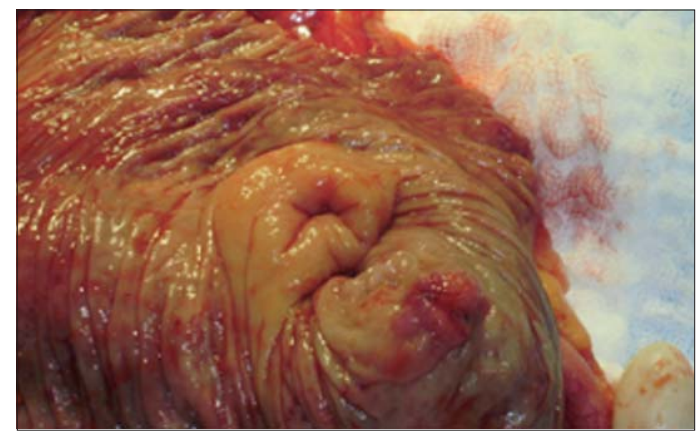

Figura 8. Pieza quirúrgica abierta. 
realiza la sección y ligadura de los vasos ileocólicos con tijeras y Vicryl ${ }^{\mathrm{TM}} 2.0$ respectivamente, el resto del mesocolon derecho se diseca con disector Enseal (EnSeal ${ }^{\mathrm{TM}}$ Laparoscopic Tissue Sealing Device, SurgRx, Ethicon) hasta el mesocolon transverso medio. Se posicionan los cabos de íleon y de colon transverso paralelos y se realiza una anastomosis íleo-transversa latero-lateral (terminal funcional) con corchetera lineal de $75 \mathrm{~mm}$ (carga azul). Posteriormente se seccionan los cabos distales de íleon y de colon transverso con una segunda carga, se extrae la pieza operatoria y se procede a reforzar las líneas de grapas con sutura continua de Vycril ${ }^{\mathrm{TM}}$ 3.0. Se reintroduce al abdomen el intestino remanente y se reinserta el puerto umbilical para visualizar y realizar el aseo de la cavidad abdominal con solución fisiológica tibia comprobándose una buena hemostasia. Se retira el puerto umbilical $\mathrm{y}$ se procede al cierre de la pared abdominal. Se cierra la aponeurosis con sutura discontinua de PDS $^{\text {TM }} 1$ y piel con Monocryl ${ }^{\mathrm{TM}} 4-0$ subcuticular (Figuras 6 y 7 ).

\section{Resultados}

La cirugía se llevó a cabo sin incidentes, el sangrado fue menor de $50 \mathrm{ml} \mathrm{y} \mathrm{el} \mathrm{tiempo} \mathrm{operatorio} \mathrm{de}$ 171 minutos. El paciente no presentó complicaciones intra ni postoperatorias. El primer día postoperatorio presentó un EVA de 0 por lo que se retiraron los opiáceos y se manejó sólo con antiinflamatorios no esteroidales endovenosos. Deambulación desde el primer día. Fue realimentado al día siguiente de la cirugía con $1.000 \mathrm{cc}$ de régimen hídrico con buena tolerancia, el segundo y el tercer día se incrementó el aporte oral a dieta líquida y liviana respectivamente con excelente tolerancia oral. Recuperó tránsito de gases y deposiciones al tercer día. Es dado de alta el cuarto día postoperatorio en buenas condiciones.

La biopsia de la pieza quirúrgica reportó una lesión exofítica cecal de $1,5 \times 1 \mathrm{~cm}$ de superficie (Figura 8), márgenes quirúrgicos a $14 \mathrm{~cm}$ de límite distal y a $7 \mathrm{~cm}$ del proximal. Histológicamente corresponde a un adenocarcinoma tubular moderadamente diferenciado, que invade hasta la submucosa (nivel SM3); Fueron analizados 30 ganglios, negativos para malignidad.

\section{Discusión}

Al igual que en las recientes publicaciones de Remzi ${ }^{2}$ y Bucher 6 , nuestro reporte presenta una hemicolectomía derecha laparoscópica utilizando sólo un puerto umbilical por el que se realiza toda la disección. Se usó una pinza angulada (Roti- culator $^{\mathrm{TM}}$ Endo Grasp ${ }^{\mathrm{TM}} 5 \mathrm{~mm}$ W/Spin Lock) y otros elementos de disección de uso habitual en cirugía laparoscópica. El procedimiento resultó sin incidentes y el paciente presentó una evolución favorable sin complicaciones posteriores. Este caso en particular difiere de lo reportado previamente en la literatura en que se trata de un tumor maligno de colon con histología confirmada.

Se tomó la decisión de llevar a cabo este procedimiento debido a que las condiciones del paciente, sano, no obeso y con un tumor pequeño e inicial, permitían realizar este tipo de cirugía sin mayor dificultad respetando todos los principios quirúrgicos oncológicos. En este caso la única diferencia con respecto a la hemicolectomía derecha laparoscópica convencional es que toda la disección se realiza a través del acceso umbilical único, evitando el uso de punciones múltiples.

La cirugía demostró seguir los principios oncológicos. Prueba de esto, es que la pieza quirúrgica revela un tumor con compromiso hasta la submucosa, con un número suficiente de ganglios (30 ganglios negativos para malignidad) y con bordes no comprometidos, lo que constituye un procedimiento quirúrgico con intención curativa. Además, corresponde a una lesión no susceptible de resección endoscópica dado que compromete la totalidad de la submucosa ( $\operatorname{sm} 3$ de la clasificación de $\mathrm{Kudo}^{9}$ ).

Respecto a las ventajas que puede ofrecer este abordaje, concordamos con otros autores en que además de un beneficio cosmético, podría disminuir la morbilidad de la introducción de otros trócares en la pared abdominal (eventraciones, sangrado y dolor postoperatorio) en comparación con la cirugía laparoscópica convencional ${ }^{2,6,8}$, pero es necesario acumular más experiencia para poder establecerlo con seguridad.

Otra potencial ventaja podría ser la utilización de esta técnica del puerto único para la inserción de trócares e instrumental auxiliar adicional en un procedimiento laparoscópico convencional con difícil disección en una zona puntual (recto bajo, ángulo esplénico), aprovechando así el mismo acceso umbilical y evitando más incisiones en la pared abdominal. Lo anterior podría impedir incluso una eventual conversión.

En nuestra opinión, con el desarrollo de nuevo instrumental y el continuo entrenamiento en cirugía laparoscópica, esta técnica podría depurarse y estar al alcance de un mayor número de cirujanos con interés en patología colorrectal. Si bien este procedimiento es hasta ahora más oneroso debido a su reciente implementación y al instrumental requerido, es probable que en un futuro cercano estos costos no sean de gran magnitud dado que los 
dispositivos no cuentan con gran complejidad mecánica ni con materiales costosos, además, con la mayor experiencia y habilidad adquirida se podría disminuir el número de ayudantes (operan sólo 2 cirujanos orientados en la misma dirección), obviando la presencia del segundo ayudante casi siempre presente en la cirugía laparoscópica de colon y recto. Si esto se cumpliera, disminuirían considerablemente los costos totales, reduciendo el personal médico necesario y a la vez realizando un procedimiento mínimamente invasivo satisfaciendo así la preferencia de los pacientes por esta modalidad quirúrgica.

Hacemos hincapié en que en esta etapa inicial se debe realizar una selección cuidadosa del paciente y se deben tomar las medidas necesarias para que el procedimiento se lleve a cabo en forma segura y oncológicamente correcta, llegando a la inserción de trócares auxiliares utilizados en cirugía laparoscópica "convencional" e incluso a la conversión si es que fuera necesario.

\section{Conclusión}

La realización de una hemicolectomía derecha laparoscópica con puerto único es factible y debería ser segura desde el punto de vista oncológico en equipos con experiencia en cirugía laparoscópica de colon. Además de las ventajas cosméticas reportadas, se podría disminuir la morbilidad de la pared abdominal, los costos totales quirúrgicos $\mathrm{y}$, eventualmente, las tasas de conversión. Por el momento constituye un procedimiento nuevo que requiere precaución en la selección de los pacientes $\mathrm{y}$ en el procedimiento a realizar.

\section{Referencias}

1. G Martel, R Boushey. Laparoscopic Colon Surgery: Past, Present and Future. Surg Clin North Am 2006; 86: 867-897.

2. F Remzi, Kirat H, J Kaouk, D Geisler. Single-port laparoscopy in colorectal surgery. Colorectal Dis 2008; 10: $823-836$.

3. Lacy AM, García-Valdecasas JC, Delgado S, Taurá P, Piqué J. Laparoscopy-assisted colectomy versus open colectomy for treatment of non-metastatic colon cancer; a randomized trial. Lancet 2002; 359: 2224 2229.

4. The Clinical Outcomes of Surgical Therapy Study Group. A comparison of laparoscopically assisted and open colectomy for colon cancer. N Engl J Med 2004; 350: 2050-2059.

5. Rosman AS, Melis M, Fichera A. Metaanalysis of trials comparing laparoscopic and open surgery for Crohn's disease. Surg Endosc 2005; 19: 1549-1555.

6. Bucher P, Pugin E, Morel P. Single port-access laparoscopic right hemicolectomy. Int J Colorectal Disease 2008; 23: 1013-1016.

7. Wilson J, Dogiparthi K, Hebblethwaite N, Clarke M. Laparoscopic right hemicolectomy with posterior colpotomy for transvaginal specimen retrieval. Colorectal Dis 2007; 9: 662.

8. Cuesta M, Berends F, Veenhof A. The "invisible cholecystectomy": a transumbilical laparoscopic operation without a scar. Surg Endosc 2008; 22: 12111213.

9. Kudo S. Endoscopic mucosal resection of flat and depressed types of early colorectal cancer. Endoscopy 1993; 25: 455-461. 